\section{La fusión de la gerencia humanista y el aprendizaje organizacional producen organizaciones sostenibles y de excelencia}

The Fusion of Humanistic Management and Drganizational Learning Create Sustainable and High Quality Drganizations

Une gestion humaniste et un apprentissage organisationne/ créent des arganisations durables et d'excellence

A fusão da gerencia humanista $E$ a aprendizagem organizacional produzem organizaçũes sustentáveis e de excelência
FECHA DE RECEPCIÓN: 10 de enero FECHA DE APROBACIÓN: 2 de febrero Pp. 74-91

Carlos Largacha-Martínez* Adela Johanna Pinzón** Elizabeth León Velásquez***

sustentaves ede excelencia
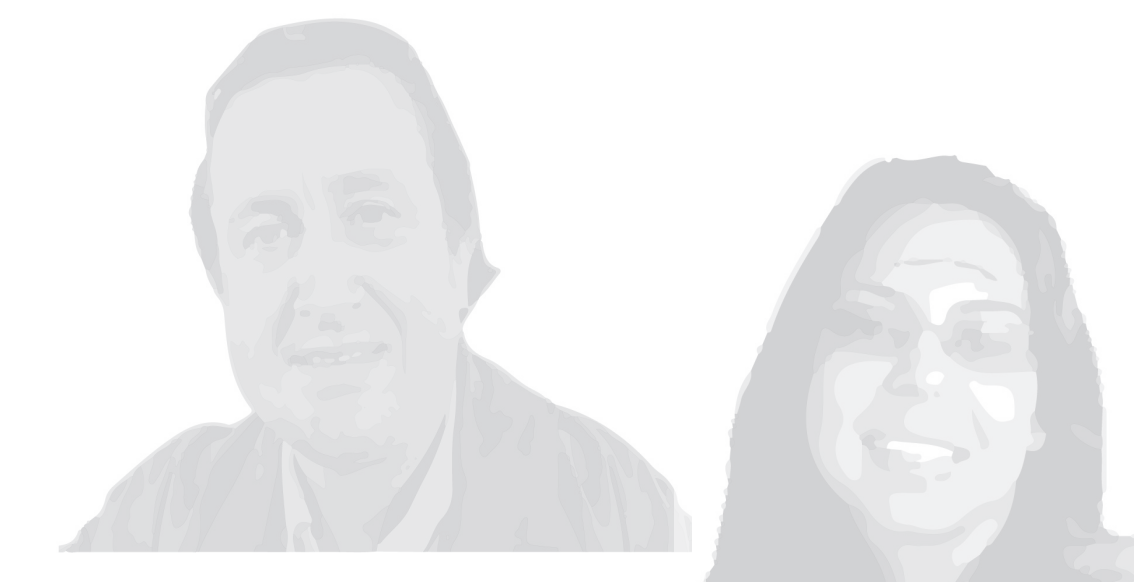


\section{RESUMEN}

En la búsqueda de mejores resultados que apalanquen la sostenibilidad empresarial, se han desarrollado diversas metodologías enfocadas a la eficiencia y la productividad. Siguiendo esta línea, las corrientes de Gestión del Talento Humano buscan trabajadores altamente productivos y tener bajas tasas de rotación de personal (Bruce, 2006; Wu \& Lee, 2001; Fisher, 2000; Lyons, 2006). Sin embargo, la mayoría de estas aproximaciones no trascienden la versión reduccionista del mercado que ve a las empresas sólo como productoras de bienes/ servicios (Schumacher, 1975), ni los paradigmas gerenciales que generan estructuras contrarias a la gestión del recurso humano (Maslow, 1968; Berger y Luckmann, 1967; Senge et.al., 1994). La Gerencia Humanista es la excepción, ofrece otra perspectiva gerencial desde la concepción de las personas en la empresa, no como recursos sino como lo que son, seres humanos. Sus principios se centran en la alteridad, las no-ideologías y las obligaciones sociales organizacionales (Largacha-Martínez, 2010b). En este artículo, se presentan los resultados de la investigación cualitativa de siete empresas, coordinada por la Red de Gerencia Humanista (Von Kimakowitz, et. al, 2011) y se analizan con relación a la excelencia y aprendizaje organizacional.

\section{ABSTRACT}

Looking for the best results that support company sustainability, diverse methodologies focusing on productivity and efficiency have been developed. Under this perspective, the trends of Human Talent Management look for high productive workers and to have low rates of personnel rotation (Bruce, 2006; Wu y Lee, 2001; Fisher, 2000; Lyons, 2006). However, most of these trends do not surpass the reductionist version of the market which only consider a company as a producer of goods or services (Schumacher, 1975), or the managerial paradigms that create structures opposing human resource management (Maslow, 1968; Berger y Luckmann, 1967; Senge et.al., 1994). The Humanistic Management is an exception as it offers another managerial option based on the existing people in the company, not seen as resources but what they really are: human beings. Its principles focus on alteration, non-ideologies and organizational social obligations

(Largacha-Martinez, 2010b). In this paper, the outcomes of a qualitative study in seven companies coordinated by the Humanistic Management Network are presented (Von Kimakowitz, et. al, 2011), analyzing them from the perspective of excellence and organizational learning.

\section{Palabras clave}

Gerencia humanista Aprendizaje organizacional Sostenibilidad

Recursos humanos

\section{Key words}

Humanistic management Organizational learning

Sustainability

Human resources 


\section{RESUMÉÉ}

Diverses méthodologies axées sur l'efficacité et la productivité entrepreneuriale ont été développées lors de recherches s'appuyant sur la viabilité de l'entreprise. Dans la littérature, les différents courants de gestion des ressources humaines insistent sur la recherche de collaborateurs hautement productifs et sur la nécessité d'obtenir de faibles taux de roulement du personnel (Bruce, 2006 ; Wu et Lee, 2001 ; Fisher, 2000 ; Lyons, 2006).

Cependant, la plupart de ces approches ne transforment pas la version réductionniste du marché qui considère les entreprises comme de simples producteurs de biens et services (Schumacher, 1975) ni les paradigmes des instances dirigeantes qui génèrent des structures contraires à la gestion des ressources humaines (Maslow, 1968; Berger et Luckmann, 1967 ; Senge et al., 1994). La Gestion Humaniste reste l'exception qui offre une perspective managériale différente de la conception du personnel de l'entreprise, qui n'est pas traité comme une ressource, mais comme des êtres humains. Les principes de la Gestion Humaniste se centrent sur l'altérité, la non-idéologie et les obligations sociales organisationnelles (Largacha-Martinez, 2010 b). Cet article présente les résultats d'une recherche qualitative effectuée dans sept entreprises et coordonnée par le Réseau de Gestion Humaniste (Von Kimakowitz, et. al, 2011) qui se centre sur le rapport à l'excellence et à l'apprentissage organisationnel.

\section{RESUMO}

Na procura dos melhores resultados que influenciem a sustentabilidade empresarial se desenvolveram diversas metodologias enfocadas a eficiência e a produtividade. Seguindo essa linha, as correntes de Gestão do Talento Humano procuram trabalhadores altamente produtivos e ter baixas taxas de rotação de funcionários. (Bruce, 2006; Wu e Lee, 2001; Fisher, 2000; Lyons, 2006). No entanto, a maioria destas aproximações não transcendem a versão reducionista do mercado que vê as empresas somente como produtoras de bens/serviços (Schumacher, 1975), nem os paradigmas gerencias que geram estruturas contrárias a gestão do recurso humano (Maslow, 1968; Berger e Luckmann, 1967; Senge et.al., 1994). A Gerência Humanista é a exceção, oferece outra perspectiva gerencial desde a concepção das pessoas na empresa, não como recursos senão como o que são, seres humanos. Seus princípios se centram na alteridade, as não-ideologias e as obrigações sociais organizacionais (Largacha-Martínez, 2010b). Neste artigo se apresentam os resultados da pesquisa científica qualitativa de sete empresas coordenada pela Rede de Gerência Humanista (Von Kimakowitz, et. al, 2011) e se analisam com relação a excelência e aprendizagem organizacional.

\section{Mots clefs}

Apprentissage organisationnel Gestion humaniste

Ressources humaines

Développement durable

\section{Palavras-chave}

Gerência humanista Aprendizagem organizacional Sustentabilidade Recursos humanos 


\section{INTRODUCCIÓN}

$\mathbf{E}$ n los círculos académicos y de gestión empresarial se ha abordado el tema de la sostenibilidad y perdurabilidad de las empresas, tratando de responder a las preguntas ¿Por qué algunas empresas logran sostenerse en el tiempo y otras no? ¿Por qué algunas han logrado seguir vigentes con resultados destacados y otras no lo consiguen? Con relación a estas discusiones, Rivera (2012) presenta un recuento de los diferentes autores que según él desde los años 80 , han venido realizando estudios para dar respuesta a dichos cuestionamientos. El resultado ha sido el desarrollo de diferentes métodos y teorías que tratan de develar el secreto del éxito empresarial.

En esta línea, Jim Collins, uno de los autores más reconocidos en gestión empresarial, realizó una amplia investigación en 22 empresas en las que llevó a cabo un análisis de sus características tratando de encontrar patrones en común (Collins, 2001). Al leer su obra, se hace visible la triste noticia de que la teoría taylorista sigue imperando en la mayoría de las empresas. $Y$ es una triste noticia porque Herzberg $(1966 ;$ 2003) mostró en los años 70, que los motivadores extrínsecos, versión reencauchada de la Teoría $X$, no generan un buen ambiente laboral y mucho menos un desarrollo a escala humana de las organizaciones y de sus trabajadores. Herzberg (2003, pp. 56-58), tomó los mejores 12 estudios sobre estos temas en donde les preguntaron a 1.685 empleados de diversos sectores y posiciones ${ }^{1}$, qué factores/eventos en la empresa generaban desmotivación (higiénicos/ intrínsecos o extrínsecos) y cuáles promovían una alta motivación (intrínsecos). Los resultados demuestran lo anteriormente descrito. Los factores extrínsecos generan el $69 \%$ de la desmotivación mientras que los intrínsecos generan, o coadyuvan a generar $81 \%$ de la motivación.
Lo interesante, y a la vez irónico, es que los resultados de Herzberg (2003) han sido mostrados y demostrados una y otra vez por estudios, desde los que Dennison y Mayo realizaron en los albores del Siglo XX (Bruce, 2006), hasta las conclusiones de Senge (2005), Collins (2001), Covey (2005), Wheatley (1992), Pérez et al. (2009), entre muchos otros ¿Existe alguna razón o forma de explicar esta tendencia a no implementar prácticas gerenciales que realmente promuevan el desarrollo humano profundo? Algunos autores como Choi y Murphy (2009), así como Dembinski (2010) muestran cómo la entrada en escena del modelo neoliberal en los años 80, con figuras simbólicas como Margaret Thatcher y Ronald Reagan, generaron una visión del desarrollo que ex-cluía la diversidad y los programas sociales de apoyo a las comunidades excluidas (ver Schumacher, 1975). Esta visión del mundo frenó los esfuerzos de cambio que la psicología humanista y las teorías críticas venían desarrollando desde los años 60. Esto con respecto a las fuerzas macroeconómicas, sin embargo, los modelos mentales socialmente construidos tienen un peso enorme en evitar el cambio como Senge $(1994,2005)$ lo muestra en sus obras.

Frente a este aspecto, Berger y Luckmann (1969) mostraron que el proceso de construcción social de la realidad tiende a la objetivación e institucionalización de la verdad, generando identidades atadas a dicha realidad, las cuales están encarceladas en la presión social debido a la legitimidad que produce dicha objetivación; el resultado final es una aversión a la exploración por fuera de la zona normal de las ideologías resultantes por parte de gerentes y líderes empresariales. Es decir, como lo describen intelectuales como Sanz de Santamaría (2010) y Craven (2010), es el sentirse vulnerable. Vulnerable a dejar el poder, miedo a no tener el control, lo cual es uno de los

\footnotetext{
1 "Los empleados, estudiados en 12 investigaciones diferentes, incluían supervisores de nivel inferior, mujeres profesionales, administradores agrícolas, personas a punto de retirarse de puestos directivos, personal de mantenimiento de hospitales, supervisores de producción, enfermeras, manipuladores de alimentos, oficiales militares, ingenieros, científicos, amas de casa, maestros, técnicos, ensambladoras femeninas, contadores, capataces finlandeses e ingenieros húngaros". Para ampliar esta información, consultar a Herzberg.
} 
principales factores que impiden el desarrollo de prácticas gerenciales centradas en el empoderamiento del trabajador (Largacha-Martínez, 2010a).

Es importante anotar que dentro de las conversaciones que se han dado en cuanto a la clave del éxito empresarial, la mayoría de ellas han girado entorno a la eficiencia y productividad en el uso de los recursos. El resultado ha sido la definición de métodos o corrientes de gestión que buscan minimizar los costos de operación, optimizar al máximo el uso de los recursos y mejorar la satisfacción de los clientes, lo que redunde en las utilidades de la empresa como fin último. Es así como nacen las corrientes de calidad, que inicialmente eran enfocadas al producto, luego se inclinaron hacia cómo se hacían estos productos, es decir, los procesos y mas adelante, se orientó hacia la gestión organizacional (Miranda, Chamorro \& Rubio, 2007).

Esta evolución en las conversaciones, ha resultado en que en la actualidad, la calidad trascienda a la excelencia. Es decir, la calidad de producto y procesos ya han dejado de ser un tema de preocupación, dado que ya no es un factor competitivo, en cambio están enfocando sus recursos y esfuerzos en lograr la excelencia organizacional, que va más allá de cumplir unos requisitos y busca lograr resultados sobresalientes en la gestión de la organización. La excelencia organizacional, se basa en el entendimiento de la empresa como un todo, un sistema que a su vez hace parte de uno mayor, por lo cual la sostenibilidad de la empresa dependerá de la sostenibilidad del sistema mayor. Por lo tanto, el objetivo de la excelencia organizacional es la creación de valor para todos los Stakeholders de la empresa, en lo que la generación de aprendizaje y su integración para la mejora de la gestión de la organización es fundamental (Corporación Calidad, 2012). A este respecto, Pérez (2010) resalta la importancia de una cultura organizacional de excelencia en la obtención de resultados sobresalientes y sostenibles, ya que genera pertenencia y como consecuencia los colaboradores se sienten comprometidos con los resultados de la empresa. Estas consideraciones, de alguna forma, abren la puerta al entendimiento de aspectos suaves de la gerencia para tratar de entender los aspectos intangibles que influyen en los resultados de la empresa.

En conclusión, el objetivo de este artículo es mostrar por qué la gerencia humanista es una de las mejores formas de potenciar la energía humana de una organización, poniendo al Ser humano como el fin y no como el medio. Esto, se sustenta en los altos niveles de empoderamiento y participación y las bajas tasas de rotación observadas en las empresas investigadas (von Kimakowitz et al., 2011). Igualmente, se presentan los resultados de la investigación cualitativa (Grounded Theory) de siete empresas a nivel global que la Red de Gerencia Humanista estudió entre 2006 y 2011, identificando sus patrones generales y analizando verdadera sostenibilidad socio-humana. Finalmente, se plantea una relación entre la gerencia humanista, aprendizaje organizacional y sostenibilidad con el objetivo de mostrar, de forma empírica, que el tener modelos mentales que observen y consideren de forma diferente a los trabajadores, influye en el logro de resultados sobresalientes que apuntan a la sostenibilidad de la empresa. 


\section{MARCO TEÓRICO}

\subsection{Gerencia humanista para la sostenibilidad}

Esta relación, gerencia y humanismo, ha sido bautizada de diferentes formas, entendida dentro de múltiples paradigmas y estudiada científicamente con contradictorios resultados (Lyons, 2006). Muchas vertientes contemporáneas marcan el inicio en la teoría administrativa de Taylor (1963), o administración científica, las cuales tuvieron una bifurcación con las ideas de Mayo y Dennison en los años 20 del siglo pasado (Bruce, 2006), y de otros pioneros como McGregor, Maslow y Herzberg, por mencionar sólo algunos. La lista de nombres que trabajan este espectro de la gerencia, es larga, pero se pueden mencionar algunos: Gerencia Participativa (Wu y Lee, 2001); Gerencia Humanista (Melé, 2003; Spitzeck et. al., 2009, von Kimakowitz, 2011; Largacha-Martínez, 2010b); Gerencia de las Relaciones Humanas (Bruce, 2006); Psicología Humanista (Lyons, 2006; Cosgrove, 2007); Comportamiento Organizacional (Robbins y Judge, 2008); Gerencia Compleja o Cuántica (Wheatley, 1992); Gerencia de la Tercera Fuerza (Maslow, 1998); Organizaciones Inteligentes (Senge, 2005); Gerencia Amigable con la Familia (Hochschild, 1997); Gerencia de Grupos de Trabajo Auto-Liderados-SDWT (Fisher, 2000).

Para entender los orígenes de estas corrientes de pensamiento, casi siempre se habla del estudio en la Planta de Hawthorne como el inicio de la Gerencia Participativa. Bruce (2006:177-178), sin embargo, coincide con otros historiadores en mostrar que esta visión hace parte de una fábula construida alrededor de los Hawthorne Studies. Independientemente de quién o quiénes lo comenzaron, lo que es relevante aquí es la entrada del Ser humano sentipensante a la escena industrial. Dentro de la revisión que Bruce (2006) realiza, se encuentran temas transversales a la Gerencia Participativa, como que se "debe reconocer la singularidad de los individuos... [así como] sus aspiraciones" (Bruce, 2006, p. 180), también la preponderancia de la colaboración por encima de la competencia, elemento descubierto por Mayo (Bruce, 2006, p. 182), y los temas de trabajo en equipo y sentido de pertenencia, como lo aseveraba Denninson para disparar la productividad y la motivación de los trabajadores (Bruce, 2006:184). Todos estos temas se entrelazaban para mostrar al trabajador como un ser complejo y a la organización como un sistema social, como lo mostraron Roethlisberger y Dickson (citado por Bruce, 2006, p. 188).

Desde la perspectiva de la participación del empleado en el puesto de trabajo, Coch y French "son considerados los pioneros" de este campo (Wu y Lee, 2001, p. 828). Lo que analizan Wu y Lee (2001, p. 830, es que la "participación en la toma de decisiones empresariales aumenta el compromiso del empleado, reduce la alienación y la resistencia al cambio, y aumenta la confianza entre el empleado y el empleador", lo cual está íntimamente atado al clima organizacional. Es decir, cualquier aproximación que una Gerencia Participativa quiera mantener, tiene que tener en cuenta el clima de la empresa, y por ende, los estilos de liderazgo, así como la cultura organizacional. Lo que sorprende es que las ideas de Mayo, Denninson, Coch, French y otros, fueron presentadas hace casi un siglo. Las últimas tendencias en Coaching o en aprendizaje organizacional tratan los mismos temas. Es decir, desde la perspectiva teórica o de las ideas, bastante ya se había dicho hace casi cien años.

Claro, el pensamiento sistémico de Senge (2005) o la empresa vista como una comunidad de personas de Melé (2003), representan propuestas episte-mológicamente muy avanzadas con respecto a la Gerencia Participativa o de Relaciones Humanas propuestas en la primera mitad del siglo XX. Empresas con propuestas de avanzada como AES, DM, Cascades, Semco, tienen índices de satisfacción muy altos en comparación con otras empresas (ver Von Kimakowitz, et.al. 2010). Resumiendo, las propuestas teóricas y prácticas de Gerencia del Recurso Humano, Gerencia Participativa, entre otras, son necesarias más no suficientes. Allí radica la importancia de la Gerencia Humanista y el valor de este escrito. 


\subsection{Sostenibilidad corporativa}

Las prácticas de gestión empresarial han evolucionado a través del tiempo condicionadas en gran parte a las exigencias normativas y de mercado, que las empresas han tenido que enfrentar. Es conocido que inicialmente

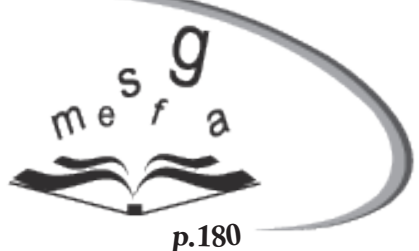
las empresas se concentraron en el cumplimiento normativo y luego trabajaron en la prevención de la contaminación con iniciativas como la producción limpia, donde se empezó a desarrollar el concepto de ecoeficiencia que incluye indicadores económicos y ambientales útiles para el desarrollo empresarial. Sin embargo, estas prácticas no son suficientes para lograr un desarrollo sostenible, además de los aspectos económicos y ambientales, se deben considerar los aspectos sociales, pues el mayor obstáculo de la sostenibilidad es la pobreza y la desigualdad social que enfrenta nuestra sociedad actual.

En 1992, en la cumbre de la tierra en Río, se evidenció aún más la preocupación por el impacto de las empresas sobre el medio ambiente y el desarrollo sostenible; a partir de esta cumbre, se inició el desarrollo de algunas iniciativas para la gestión ambiental empresarial como el Eco-Management and audit. Sheme (EMAS), la ISO 14001 y otras normas e iniciativas que les siguieron posteriormente y además de los aspectos ambientales y económicos de las organizaciones, incluyeron los aspectos sociales como necesarios para hablar de sostenibilidad. Siguiendo este concepto de sostenibilidad muchas empresas han sumado a sus prácticas de gestión ambiental, prácticas de responsabilidad social, adoptando numerosas iniciativas entre las cuales se pueden mencionar:

- "La carta de negocios para el desarrollo sostenible" iniciativa de la Cámara de Comercio Internacional, cuyo objetivo es promover en las empresas acciones para combatir el deterioro ambiental y la escasez de los recursos naturales. La carta está constituida por 16 principios rectores que incluyen el enfoque preventivo de los productos y servicios de las empresas que la han firmado (aprox. 2.300 empresas a nivel mundial)².

- Los objetivos del Milenio comprenden ocho propósitos de desarrollo, fijados en el año 2000 por 189 países miembros de las Naciones Unidas, quienes acordaron conseguir dichos objetivos para el año 2015. Comprenden la erradicación de la pobreza y el hambre, lograr la enseñanza primaria universal, promover la igualdad de géneros, reducir la mortalidad infantil, mejorar la salud materna, combatir el VIH/SIDA y otras enfermedades, garantizar la sostenibilidad del medio ambiente y fomentar la asociatividad mundial para el desarrollo.

- El Pacto Mundial, el cual es una iniciativa voluntaria por la cual las empresas se comprometen a focalizar sus estrategias y acciones en diez principios que fueron universalmente aceptados en cuatro temáticas principales: derechos humanos, estándares laborales, medio ambiente y anticorrupción. Es una de las iniciativas ciudadanas más grandes del mundo, con casi 12.000 participantes en 145 países $^{3}$.

- Norma ISO 26000: Guía sobre Responsabilidad Social Empresaria. Es una norma que permite a las organizaciones establecer, mantener y mejorar sus acciones de responsabilidad social con sus Stakeholders, así como a comunicar los compromisos de la organización con respecto a dichas acciones.

- "La Visión 2050: una nueva Agenda para los Negocios" del World Business Council for Sustainable Development, la cual propone acciones a implementar por las empresas y gobiernos para afrontar los retos que en materia de explotación de recursos y deterioro ambiental, tiene nuestra sociedad actual.

\footnotetext{
2 Para ampliar esta información, consultar: http://www.iccspain.org

${ }_{3}^{3}$ Para ampliar esta información, consultar: http://www.unglobalcompact.org/languages/spanish.
} 
Las iniciativas, en cuanto a normatividad y acuerdos voluntarios, sin duda han favorecido la realización de prácticas empresariales hacia una mejor gestión ambiental en los procesos productivos, disminuyendo los impactos sobre el medio ambiente y el deterioro del mismo. Algunas empresas han incluido prácticas de responsabilidad social, lo que permitirá hablar de prácticas que buscan el desarrollo sostenible de las empresas, si se considera que tienen en cuenta los aspectos económicos, ambientales y sociales relacionados con sus actividades. Sin duda, la responsabilidad de la sostenibilidad no es solo trabajo de las empresas y es necesario hacer cambios fundamentales en los individuos y en las relaciones entre ellos, para lograr un desarrollo sostenible como lo menciona Ehrenfeld (2004) en su artículo "Buscando la Sostenibilidad"4.

La Corporación Calidad, define la sostenibilidad como la "condición que garantiza que los objetivos e impactos positivos perduren en el tiempo. Se logra cuando la satisfacción de las necesidades presentes, no compromete la capacidad de las generaciones futuras para satisfacer sus propias necesidades" (Corporación Calidad, 2012, p. 55). También, se ha definido como el Triple Bottom Line o "Triple balance", sugiriendo que para la sostenibilidad debe existir generación de riqueza o largo plazo, creación de empleo y maximización de recursos, eliminando o disminuyendo la degradación ambiental (Scott, 2010).
Las anteriores definiciones e iniciativas presentadas muestran la necesidad de considerar en la sostenibilidad empresarial, no solo los procesos (uso eficiente de materiales y energía, generación de productos de menor impacto ambiental, disminución de la contaminación),

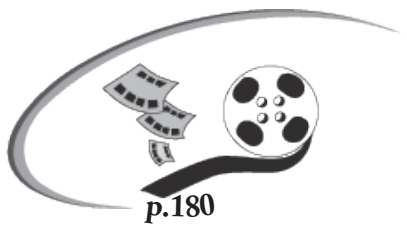
sino ir más allá, considerando los aspectos sociales relacionados con la empresa los cuales incluyen igualdad, trato justo, proveer oportunidades para sus empleados y diferentes socios de tal forma que todos contribuyan a mejorar los procesos en busca de la sostenibilidad.

\subsection{Aprendizaje organizacional}

El aprendizaje organizacional se puede entender como la tarea de tratar de identificar una relación entre excelencia organizacional y aprendizaje organizacional. Wenbin y Hongyi (2009), realizaron un estudio enfocado en identificar qué tipo de relación causal existe entre mejoramiento continuo y aprendizaje organizacional. Según sus conclusiones, existe una relación mutuamente edificante entre estos dos aspectos.

A continuación se presenta una comparación entre los diferentes enfoques de gestión de calidad empresarial (Tabla 1). 
Tabla 1. Comparación entre los enfoques de gestión de la calidad

\begin{tabular}{|c|c|c|c|c|}
\hline \multirow[t]{2}{*}{ Características } & \multicolumn{4}{|c|}{ Enfoques de la gestión de la calidad } \\
\hline & Inspección & Control & Aseguramiento & Calidad total \\
\hline Objetivo & Detección de defectos. & $\begin{array}{l}\text { Control de productos } \\
\text { y procesos. }\end{array}$ & $\begin{array}{l}\text { Organización y } \\
\text { coordinación. }\end{array}$ & $\begin{array}{l}\text { Impacto estratégico de } \\
\text { la calidad. }\end{array}$ \\
\hline $\begin{array}{l}\text { Visión de la } \\
\text { calidad }\end{array}$ & Problema a resolver. & Problema a resolver. & $\begin{array}{l}\text { Problema a resolver de } \\
\text { forma activa. }\end{array}$ & $\begin{array}{l}\text { Oportunidad para } \\
\text { alcanzar una ventaja } \\
\text { competitiva. }\end{array}$ \\
\hline Énfasis & $\begin{array}{l}\text { En el suministro informe } \\
\text { de componentes. }\end{array}$ & $\begin{array}{l}\text { En el suministro } \\
\text { uniforme de } \\
\text { componentes. }\end{array}$ & $\begin{array}{l}\text { En la totalidad de la } \\
\text { cadena de valor } \\
\text { añadido. }\end{array}$ & $\begin{array}{l}\text { En el mercado y en las } \\
\text { necesidades del cliente. }\end{array}$ \\
\hline Métodos & $\begin{array}{l}\text { Fijación de estándares y } \\
\text { medición. }\end{array}$ & $\begin{array}{l}\text { Muestreo y técnicas } \\
\text { estadísticas. }\end{array}$ & $\begin{array}{l}\text { Programas y sistemas. } \\
\text { Planificación estratégica. }\end{array}$ & Planificación estratégica. \\
\hline Responsabilidad & $\begin{array}{l}\text { Departamento de } \\
\text { inspección. }\end{array}$ & $\begin{array}{l}\text { Departamento de } \\
\text { producción. }\end{array}$ & $\begin{array}{l}\text { Todos los } \\
\text { departamentos. }\end{array}$ & $\begin{array}{l}\text { La dirección de forma } \\
\text { activa, y con ella, el } \\
\text { resto de la organización. }\end{array}$ \\
\hline Orientación & Producto. & Proceso. & Sistema. & Personas. \\
\hline Enfoque & La calidad se comprueba. & $\begin{array}{l}\text { La calidad se } \\
\text { comprueba. }\end{array}$ & La calidad se produce. & La calidad se gestiona. \\
\hline
\end{tabular}

Fuente. Miranda, Chamorro \& Rubio, 2007; tomado de Benavides y Quintana, 2003.

Tabla 2. La organización que aprende vs. la organización tradicional

\begin{tabular}{|l|l|l|}
\hline \multicolumn{1}{|c|}{ Organización tradicional } & \multicolumn{1}{c|}{ Organización que aprende } \\
Actitud ante los cambios. & Si fuciona, no lo cambies. & Si no lo cambias, dejará de funcionar. \\
Actitud ante las ideas nuevas. & Si lo inventamos o reinventamos aquí, \\
¿Quién es responsable de la innovación? & Si no lo inventamos aquí, recházalo. & recházalo. \\
Temor principal. & Cometer errores. & Todos los miembros de la organización. \\
Ventaja competitiva. & Productos y servicios. & No aprender; no adaptarse. \\
Trabajo del gerente. & Controlar a los demás. & y experiencia. \\
& & Facultar a los demás. \\
\hline
\end{tabular}

Fuente. Robbins \& Coulter, 2005.

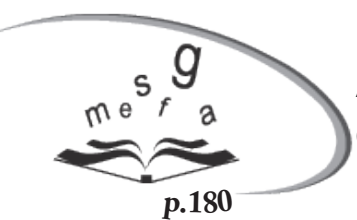

Muhamad, Cory, Garvvare y Ahmad (2011), analizan la correlación entre excelencia empresarial y sostenibilidad, y aunque mencionan que un modelo de excelencia per se no es suficiente para que la empresa adopte prácticas y tome en cuenta la sostenibilidad en todas sus decisiones, si es una plataforma que permite que las empresas avancen hacia ese camino. 


\section{MARCO METODOLÓGICO}

$\mathbf{L}$ a investigación realizada en este proyecto es de tipo cualitativa, en la que se aplicó un análisis de contenido y muestreo teórico (content analysis \& grounded-theory) de seis empresas a nivel global, que la Red de Gerencia Humanista (Humanistic Management Network) estudió entre 2006 y 2009, documentando los patrones generales de dichas empresas en 14 casos, con el fin de identificar los patrones que generan verdadera sostenibilidad en dichas empresas.
Para realizar esta investigación, se utilizó el Software de análisis de contenido MaxQDA v.104, el cual permite contabilizar la ocurrencia inductiva de textos para cada una de las empresas, además de poder realizar análisis cruzados de las ocurrencias de los factores. Para contextualizar el estudio y el análisis, a continuación se listan las empresas investigadas, ubicación, tamaño y sector industrial y se muestra cómo se realizó el análisis de contenido y el muestreo teórico (Tabla 3 y Figura 1).

Tabla 3. Empresas investigadas en el presente proyecto

\begin{tabular}{|l|l|l|l|}
\hline \multicolumn{1}{|c|}{ Compañía } & \multicolumn{1}{c|}{ Ubicación } & \multicolumn{1}{c|}{ Tamaño } & \multicolumn{1}{c|}{ Sector Industrial } \\
\hline ABN Amro Banco Real & Brasil & Grande / Nacional & Servicios \\
\hline AES Corporation & Estados Unidos & Grande / Global & Bienes industriales \\
\hline Cascades Pulp and Paper & Canadá & Grande / Global & Bienes industriales \\
\hline$d m$ & Alemania & Grande / Nacional & Servicios \\
\hline Semco & Brasil & Grande / Nacional & Conglomerado \\
\hline Wainwright Bank \& Trust & Estados Unidos & Pequeña / Nacional & Servicios \\
\hline Views & Colombia & Pequeña / Nacional & Servicios \\
\hline
\end{tabular}

Fuente. Von Kimakowitz, et.al, 2011, con datos adicionales por el autor ${ }^{5}$.

Figura 1. Instrumento utilizado para el análisis de contenido y muestreo teórico

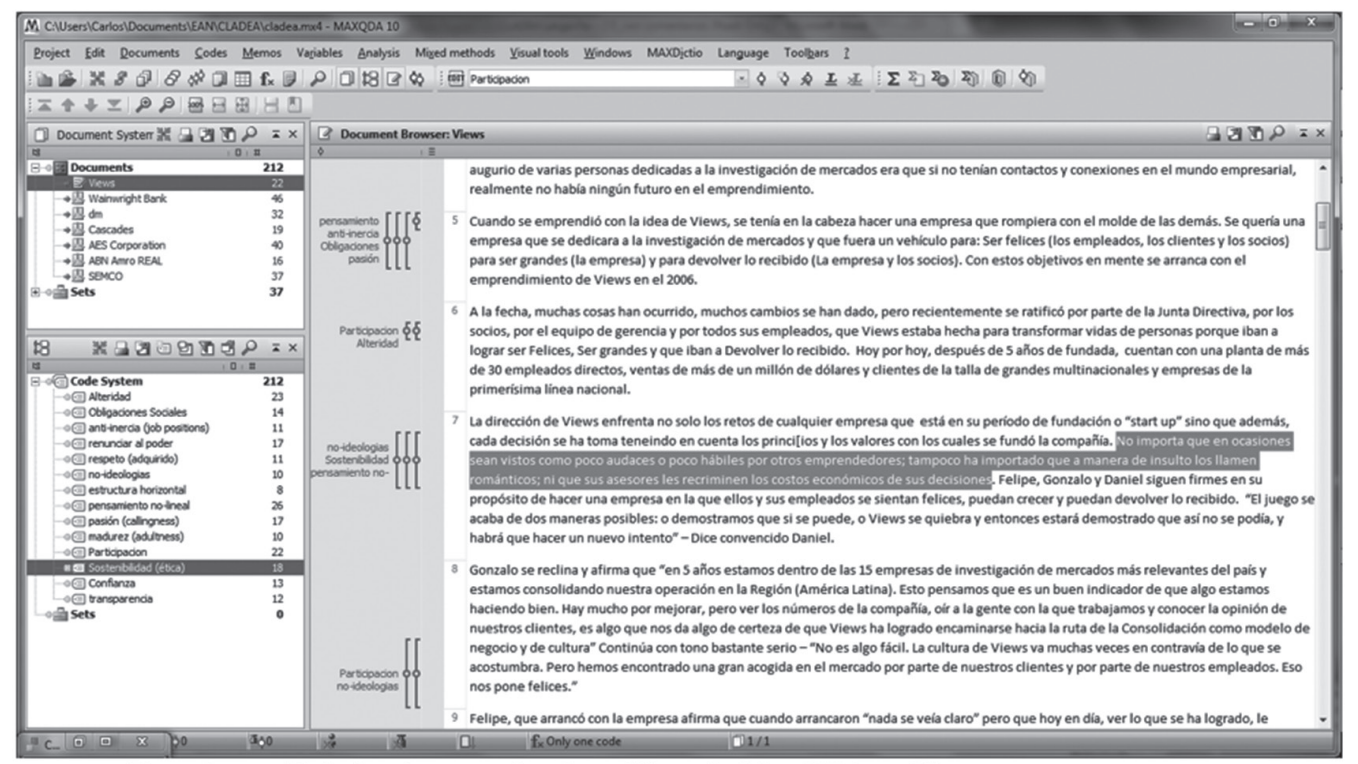

Fuente. Análisis propio de los autores, a partir de MaxQDA, v.10.

4 "MAXQDA is a state-of-the-art instrument for professional text analysis". Para conocer más sobre esta herramienta, consultar:http:// www.maxqda.com/products-. QDA es la sigla de 'Qualitative Data Analysis'.

5 La empresa colombiana Views no fue parte del estudio realizado por Von Kimakowitz, et.al. (2011), fue objeto de un estudio con las mismas características, realizado por los autores del presente artículo. 
Los datos para la investigación parten de los seis capítulos (uno por empresa), escritos en el libro "Humanistic Management in Practice" (von Kimakowitz, 2011), así como de la investigación en curso $^{6}$ en la empresa Views, para completar la muestra de siete empresas. Se realizó un análisis inductivo y de contenido para generar 14 constructos, partiendo de una de las empresas seleccionadas (SEMCO), y desde allí, se generó un proceso factorial acumulativo para las demás empresas (ver Gilgun et. al., 1992). El proceso de SEMCO se realizó a través del análisis de contenido (ver White y Marsh, 2006) de dos libros y cuatro artículos de revistas, lo cual fue complementado con ocho entrevistas telefónicas con Joao Vendramim ${ }^{7}$, durante los meses de enero a agosto de 2006. El análisis cualitativo terminó con un acumulado de coincidencias (repeticiones) con los 14 constructos realizados en la primera fase del muestreo teórico. Esto ayuda a ver aquellos que más se repiten en las otras seis empresas y de esta forma emergen los patrones más importantes.

Se muestra el primer momento de análisis realizado en SEMCO, con sus 12 constructos (variables del muestreo y análisis de contenido). De esta forma se tienen los resultados para hacer el análisis, elemento a presentar en la siguiente sección (Figura 2).

Figura 2. Constructos (codes) de SEMCO, primer nivel de análisis

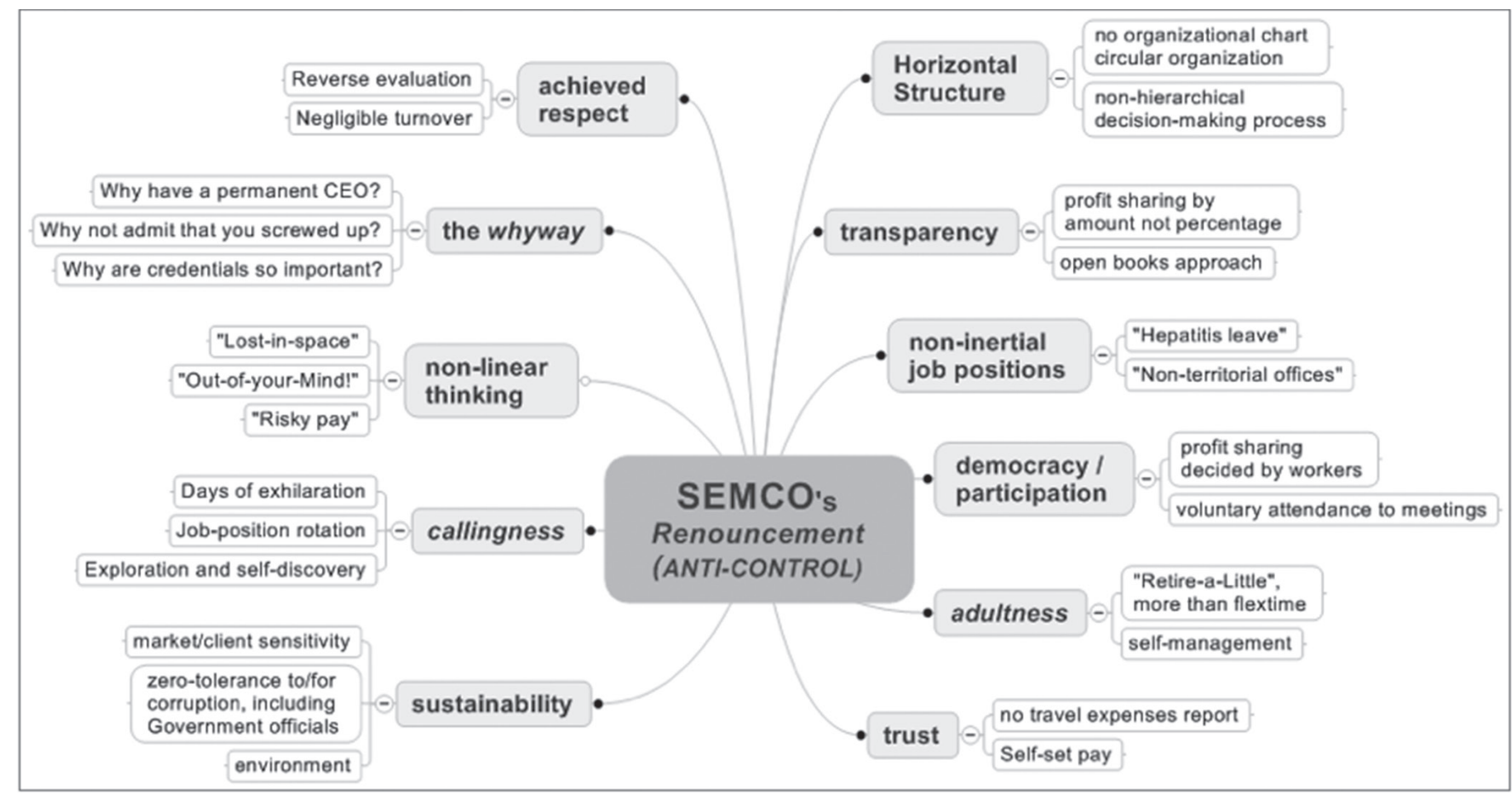

Fuente. Largacha-Martínez, 2010a.

\footnotetext{
${ }^{6}$ Dentro del grupo Tendencias en Gestión e Innovación (TGI), Categoría B de Colciencias, y en su línea Gerencia Humanista, se realizó la investigación a la empresa colombiana Views 2010-2011, gracias a fondos para investigación de la Universidad EAN.

7 Joao es consejero de Ricardo Semler, CEO de Semco y fue Vicepresidente de Tecnología y Manufactura de Semco.
} 


\section{ANÁLISIS DE DATOS}

1

continuación se presentan los resultados del segundo nivel de análisis, en donde los cia de acuerdo con el análisis de contenido realizado (Figura 3). De esta forma gráfica es más fácil ver los patrones de ocurrencia factorial de los constructos seleccionados bajo la metodología mencionada, con lo cual se potencia el análisis de las investigaciones realizadas en estas empresas.
Se observó cómo la mayoría de los constructos se presentan en la mayoría de las empresas. Esto significa que todas estas sí cumplen con los principales postulados de la Gerencia Humanista (von Kimakowitz, et.al., 2010; Largacha-Martínez, 2010b). En cuanto a las mayores frecuencias entre las siete empresas están: pensamiento no lineal (26), alteridad (23), Participación (22), sostenibilidad (18) y renunciar al poder / pasión (17). Es interesante que el constructo estructura horizontal, sea el de menos frecuencias (8). Se crearon dos nuevos constructos en el segundo nivel de análisis: alteridad y obligaciones sociales.

Figura 3. Frecuencia de constructos, segundo nivel de análisis

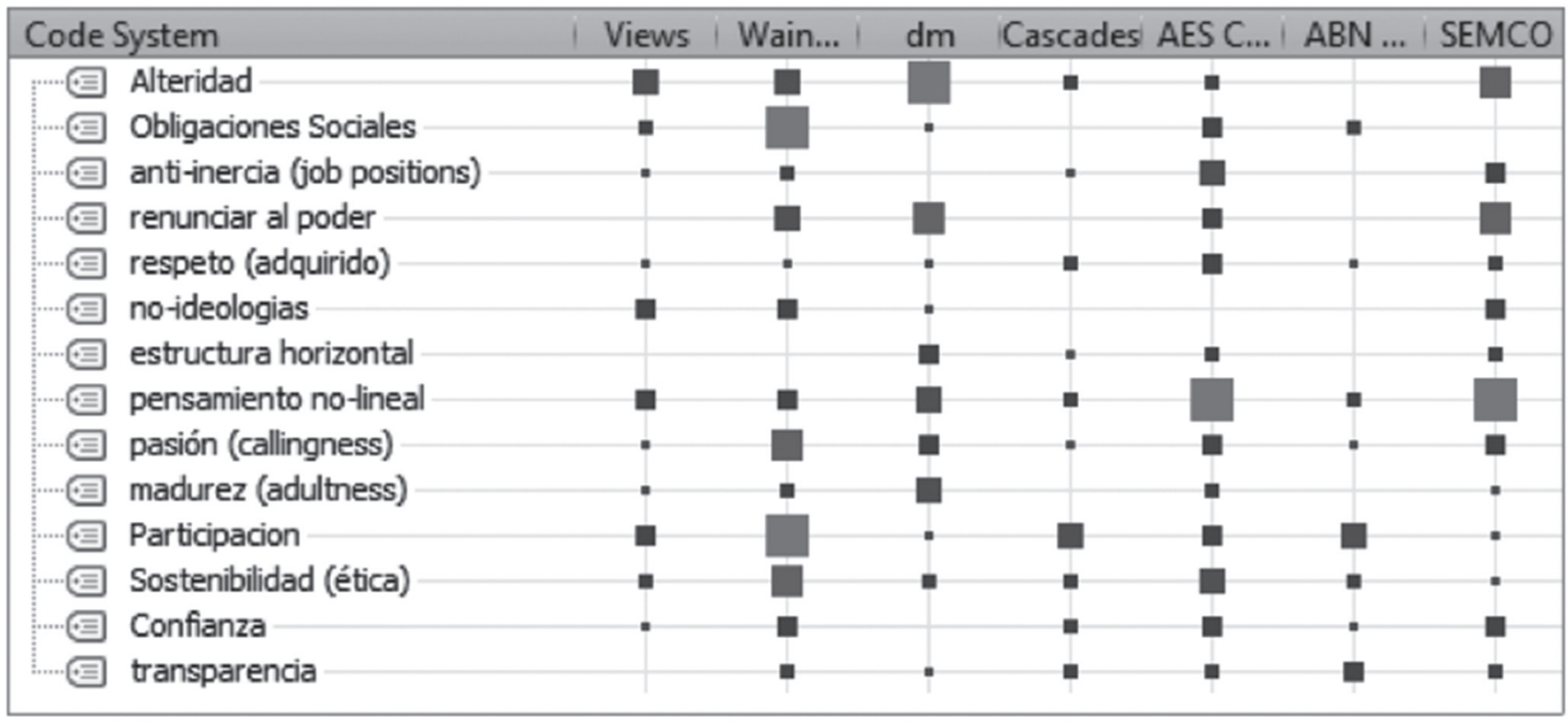

Fuente. Análisis propio de los autores, a partir de Code Matrix Browser de MaxQDA, v.10.

Adicionalmente se corrió el Code Relations Browser para conseguir la correlación entre todas las variables (Figura 4). Por ser una correlación simple, la tabla se repite diagonalmente hacia un lado. Las correlaciones más representativas son los duetos: participación/renuncia-poder (5), madurez/pasión (5), participación/pensamiento-no lineal (4). Hay muchos duetos con tres frecuencias, luego son estas tres duplas las que serán presentadas en los resultados. Es interesante ver que las mayores frecuencias totales de las correlaciones obtenidas están en: participación (31), alteridad (29), sostenibilidad (24) y renunciar al poder (21). 
Con base en lo anterior, es posible comenzar a mostrar los resultados del análisis de la investigación cualitativa desarrollada. Es importante aclarar las limitaciones de esta investigación, pues se realizó de forma «Clusterizada», comenzando por SEMCO y no por un análisis de todas las compañías para allí sí diseñar y crear los constructos. Sin embargo, esta aproximación metodológica ayudó a centrar la investigación en donde los autores tenían mayor conocimiento y tiempo de investigación, habían leído más fuentes y tenido acceso a fuentes primarias. Para futuras investigaciones se podría comenzar por el análisis de un solo nivel y no de segundo nivel como el desarrollado en este documento.

\section{Figura 4. Correlación de constructos.}

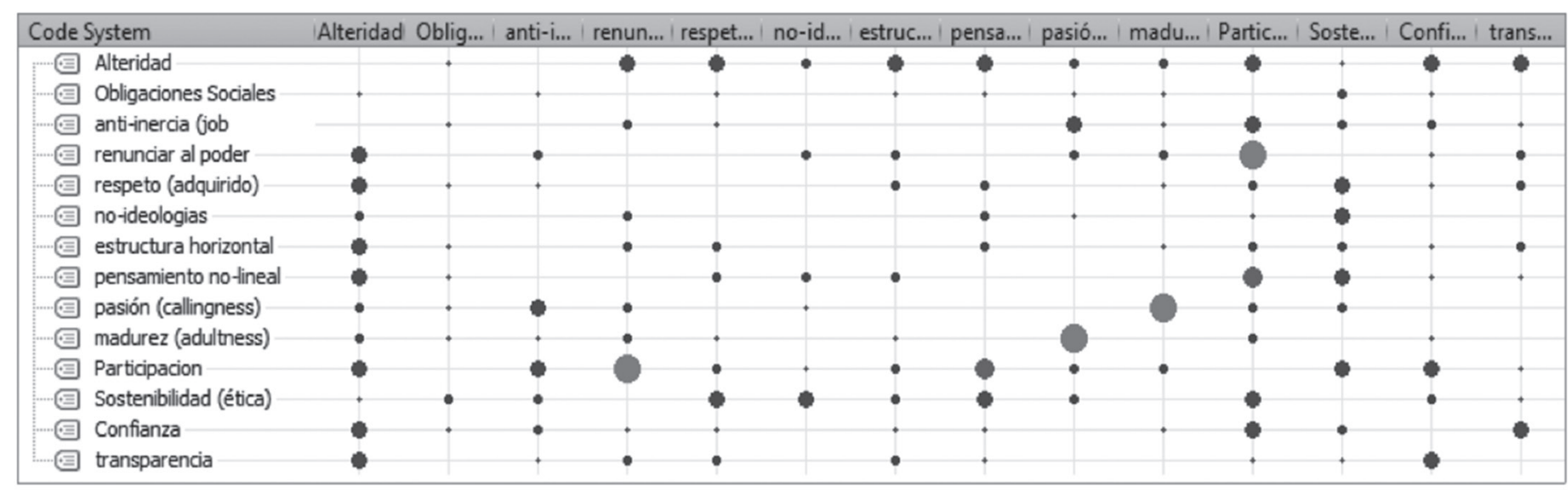

Fuente. Análisis propio de los autores, a partir de Code Relations Browser de MaxQDA, v.10.

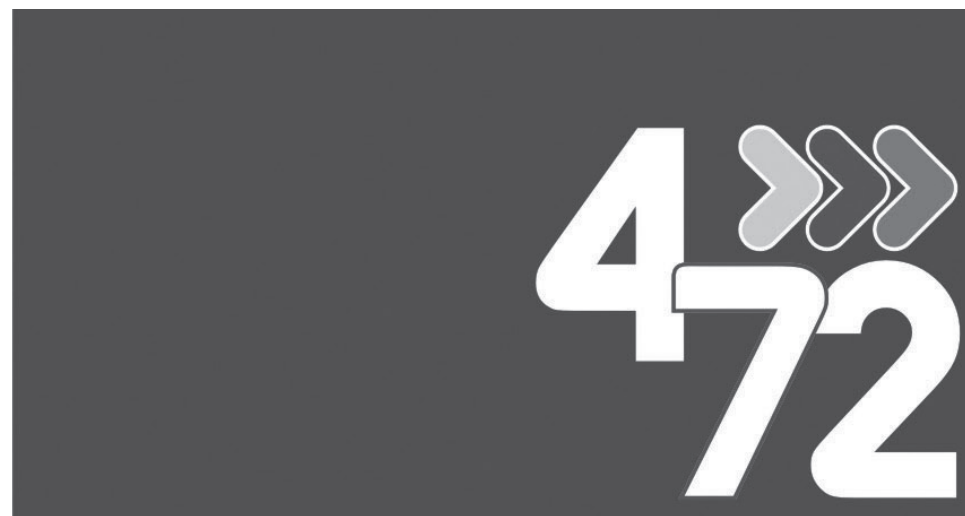

LARED POSTAL DE COLOMBIA

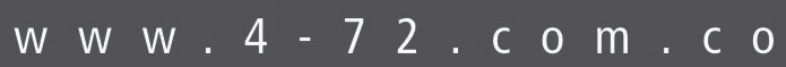

\section{S Línea de Atención al Cliente Nacional 018000111210}




\section{RESULTADOS}

L os gerentes humanistas no piensan linealmente. Es decir, piensan por «fuera de la caja». Este sería el primer resultado. Es el constructo de mayor ocurrencia con 26 veces. De ahí, la importancia de trabajar los modelos mentales de las empresas para promover el cambio. Esto, por ejemplo, no lo trabajan intencionalmente las aproximaciones tradicionales de gestión del recurso humano (Largacha-Martinez, 2010b). La dificultad radica en que como los "modelos mentales son usualmente tácitos, existen por debajo del nivel de conciencia, frecuentemente no se examinan ni se evalúan" (Senge et. al, 1994:236). Por eso, la quinta disciplina trabaja con la generación de bibliotecas de modelos mentales organizacionales, como lo hizo Bill O'Brien en su empresa Hannover Insurance (Senge, 2005). Esto exige búsqueda interior y reflexión, lo cual implica alta confianza (o baja vulnerabilidad, como ya se expuso).

Si un modelo mental es el de Command \& control, difícilmente se podrá ser humanista, pues como lo muestran los datos, alteridad es el constructo que le sigue con 23 ocurrencias. Dignificar al otro es fundamental, sería el segundo resultado, lo cual se articula muy bien con el tercer constructo, participación (22). El problema radica en que "participación consultiva", como lo define Semler (2003) sin poder tomar decisiones, no es realmente participación. Las posturas tradicionales hablan mucho de esto pero al no estar atado a libertad para la toma de decisiones y libertad para el error (que implica un modelo mental no lineal, o no tradicional, y una búsqueda por potenciar al otro), se quedan en el discurso. En estas empresas humanistas, pueden sacar de una reunión al dueño de la empresa, quitarle la oficina al Presidente de la compañía durante un viaje, no rendirle pleitesía a los dueños, y lo que demuestra que es cierto, es que lo pueden hacer "sin sentirse culpable... [y] sin pedir permiso", como lo dijo Zeca, trabajador de SEMCO cuando estuvo todo un día viendo la final de tenis del Roland Garros (Semler, 2003, p. 33). Y qué dijo a esto el CEO de SEMCO (y con esto se resume lo expuesto).
He didn't miss a match, and his work didn't suffer either. On the contrary, pursuing his hobbies or his girlfriend allows him to create balance in his life" (Ibid.). ¿Ustedes creen que Donald Trump contestaría igual? ¡Yo creo que diría 'you are fired!

Los otros tres constructos, sostenibilidad, renunciar al poder y pasión en el trabajo, complementan el espacio de la gerencia humanista. Todas estas empresas ponen de primero al Ser humano (von Kimakowitz, et. al. 2011) y tienen rentabilidades financieras superiores al promedio. A todas les importa el medio ambiente, no contaminar, no ser corruptos, por lo que son realmente sostenibles. Otro ejemplo que sirve para demostrar este resultado es el referente al banco norteamericano Wainwright cuando se dice que "Over the past 20 years Wainwright has invested $\$ 700$ million in community development loans, and not one has ever failed.... That's not only atypical; it is radically unsettling to the banking world's prevailing mindset" (Arena, 2010, p. 280).

Por limitaciones de espacio no se pueden mencionar todos los premios que estas empresas han recibido ${ }^{8}$, el buen nombre que tienen en sus países, las tasas de rotación tan bajas, entre otros muchos aspectos. En una de ellas con 3.000 empleados, durante dos años sólo se fueron de la empresa dos personas. Las citas a empleados que no se van a si les den el doble del sueldo son permanentes. Las citas a la importancia del diálogo, de la confianza, de la transparencia son muestra de que no estamos hablando de algo que necesita mucha inversión de tiempo o recursos financieros. Se necesita mucha paciencia, eso sí.

Por último mostrar que las correlaciones fundamentan aún más lo encontrado en esta investigación. Las duplas y los datos encontrados nos dicen que las empresas con gerencia humanista cuando hay participación, se renuncia al poder, al control (5). Cuando hay madurez por parte de los trabajadores, se presenta mayor pasión por lo que hacen, o viceversa (5). Y que buenos niveles de participación se dan cuando está presente el pensamiento no-lineal, la intuición y el espacio para explorar cosas atípicas (4).

\footnotetext{
8 El libro de "Humanistic Management in Practice" tiene 19 empresas estudiadas. Aquí sólo se analizan seis de estas, sin embargo, los patrones que se muestran son transversales a todas.
} 


\section{CONCLUSIONES}

¡Sí se puede! Esta es la mejor forma de concluir este artículo. Sí se le puede dar la mano al empleado, y si es bien tratado, no le va a coger el codo. Si el lector quiere conocer los detalles, se invita a leer el libro donde se realizó el análisis de contenido y el muestreo teórico. El objetivo de este documento era mostrar qué necesitan las empresas para lograr lo que otras han logrado, y qué debe realizar la gestión del recurso humano para obtenerlo. Lo que se encontró es que se debe trabajar en modelos mentales abiertos a la reflexión, pensamiento no lineal para no rechazar de tajo propuestas no lógicas, entender que el Ser humano es complejo y sensible y necesita mucho respeto, y que entre otras cosas, si el puesto de trabajo no es apasionante, difícilmente los colaboradores logran ser altamente productivos.

Views está incursionando desde su inicio, sin saberlo, en la gerencia humanista. Desde hace dos años está siendo investigada por el Dr. Carlos LargachaMartínez. Al conocer que su visión es «ser felices» (Pérez, 2010), que les dan el $20 \%$ de su tiempo de trabajo para que hagan lo que quieran, que los apoyan para que creen sus propias empresas, entre muchas otras ideas, se les comentó a los socios que eran un gran ejemplo. Sin embargo, a los cuatro años de funcionamiento, con 40 empleados y U\$1 millón de dólares en ventas al año, descubrieron que diez trabajadores los estaban robando ${ }^{9}$ desde hace un año. Llegaron a la oficina del Dr. Largacha y dijeron: "¿Qué hacemos?" Él se quedó sin palabras. Se habían realizado ya tres reuniones con todo el personal. La consultoría iba viento en popa a tal punto que después de un taller, una colaboradora que iba a renunciar no lo hizo. Ellos se aferraron a sus principios. No los despidieron. Les dieron una segunda oportunidad, sin embargo los siguieron robando. Los mantuvieron, pero les dijeron que mejor renunciaran. Sintieron miedo. Se sintieron vulnerables. Parecía que todo había sido en vano. Que sus allegados y abogados tenían razón. Que eran unos bobos, románticos e ilusos. Serendipity, esta palabra resume nuestro encuentro. Para los socios el haber encontrado que otras empresas practicaban algo llamado "gerencia humanista" les dio el empujón que necesitaban. Ojalá muchos más se arriesguen. Los socios de Views lo hicieron y concluyen: "¡Decidimos que si alguien quería volvernos a robar, lo iba a poder hacer! Pero nosotros no íbamos a tratar diferente a la gente de cómo pensábamos que estaba bien hacerlo!" (Pérez, 2010).

Concluir que son necesarias las propuestas de gestión del recurso humano, más no suficientes para lograr que el Ser humano sea el fin de las empresas, pues no cubren todos los principios de la gerencia humanista: alteridad, no-ideologías, obligaciones sociales organizacionales, respeto incondicional a la persona, ética normativa y la alineación ética-resultados.

Es importante mencionar que cuando se habla de sostenibilidad empresarial, se debe considerar con un enfoque comercial y que las acciones o estrategias de sostenibilidad deben estar encaminadas a generar valor a los accionistas, considerando las oportunidades y gestionando los riesgos que se derivan de los desarrollos económicos, ambientales y sociales; estos últimos, por supuesto, incluyendo al Ser humano que hace parte de las empresas, integrándolos y haciéndolos parte del crecimiento empresarial. La sostenibilidad promueve la innovación a través de respuestas a los nuevos retos y a las necesidades del entorno. Pero finalmente no hay que olvidar que dichas necesidades son identificadas por el Ser humano y que los procesos de innovación en respuesta a esas necesidades son iniciadas por seres humanos. Dichos procesos son más eficaces cuando hay motivación e integración de los empleados en el desarrollo empresarial.

\footnotetext{
${ }^{9}$ Montaron una empresa paralela, les quitaban clientes y trabajaban con los computadores de Views.
} 


\section{REFERENCIAS BIBLIOGRÁFICAS}

Arena, C. (2010). Wainwright Bank and Trust Case Study. Humanistic Management in Practice, Ernst von Kimakowitz, et al., editors. Palgrave McMillan, pp. 277-289.

Berger, P. \& Luckmann, T. (1966). The Social Construction of Reality. (s.c): Doubleday.

Bruce, K. (2006). Henry S. Dennison, Elton Mayo, and Human Relations historiography. Management \& Organizational History, 1(2).pp. 177-199.

Cámara de Comercio Internacional (ICC: International Chamber of Commerce) (1991). Carta de Negocios para el Desarrollo Sostenible. (s.c) (s.e).

Choi, J.; Murphy, J. (2009). Globalisation and the Prospects of Critical Reflection. (s.c): Aakar.

Collins, J. (2001). Good to Great. (s.c): HarperBusiness.

Corporación Calidad (2012). Premio Nacional a la Excelencia y la Innovación en Gestión 2012-2013: Modelo de clase mundial para la competitividad y la sostenibilidad. Recuperado de http://www.premionacionalexcelencia. org/descargas/cartilla_PNEIG_2012-2013.pdf

Cosgrove, L. (2007). Humanistic Psychology and the Contemporary Crisis of Reason. The Humanistic Psychologist, 35(1), pp. 15-25.

Covey, S. (2005). El octavo hábito: de la efectividad a la grandeza. Barcelona: Paidós.

Craven, A. (2010). State of the Art in human relations Management, charla dictada como parte de la alianza University of Incarnate Word y Universidad EAN.

Dembinski, P. (2010). Efficiency vs. Fecundity: from Relation to Transaction and ... (possibly) back. Ponencia en IESE, Barcelona. Recuperado de: http://www.iese.edu/16symposium

Ehrenfeld, (2004). Buscando la Sustentabilidad. Reflections. 5, (8).

Fisher, K. (2000). Leading Self-Directed Work Teams. (s.c): McGraw-Hill.

Herzberg, F. (1966). Work and the Nature of Man. The World Publishing Company.

Herzberg, F. (2003). One More Time: How Do You Motivate Employees?. Harvard Business Review on Motivating People, (s.c): Harvard Business Publishing.

Hochschild, A. 1(997). The Time Bind. New York: Metropolitan Books.

Kuhn, T. (1996). The Structure of Scientific Revolutions. Chicago: University Of Chicago Press. 
Largacha-Martínez, C. (2010a). Quantum Humanism as a Framework for Humanistic Management. Ponencia en IESE, Barcelona. Recuperado de: http://www.iese.edu/16symposium

Largacha-Martínez, C. (2010b). Aproximaciones a la Gerencia Humanista. Bogotá: Universidad EAN.

Largacha-Martínez, C. (2011). Quantic Humanism. E-book, Kindle edition.

Lyons, A. (2006). Recalling the Past, Creating a Future: Challenges for Humanistic Research. The Humanistic Psychologist, 34(1), 13-20.

Maslow, A. (1998). Maslow on Management. Wiley, Canada.

Melé, D. (2003). The Challenge of Humanistic Management. Journal of Business Ethics, 44, pp. 77-88.

Miranda, F; Chamorro, A. \& Rubio, S. (2007). Introducción a la gestión de la calidad. Madrid: Delta publicaciones

Pérez, D. (2010). La estrategia de Views. Charla en clase "Gerencia Humanista", Universidad EAN.

Pérez, R. (2010). Compromiso de la alta gerencia en la creación y mantenimiento de una cultura organizacional de excelencia. En Largacha-Martínez (Ed.), Aproximaciones a la Gerencia Humanista: desde lo transversalidad de lo global, lo corporativo y lo comunitario, Bogotá: Universidad EAN, pp. 143-196.

Pérez, R.; Nieto, M.; Velásquez, A.; Castellanos, G.; Garzón, M.; Vargas, H.; Alfonso, N.; Calixto, N.; Rodríguez, A.; Palacio, M.; López, L.; Vidal, M. y López, J. (2009). Modelo de modernización para la gestión de organizaciones. Bogotá, Colombia: Universidad EAN.

Rivera, H. (2012). Perdurabilidad empresarial: concepto, estudios, hallazgos. Cuadernos de Administración, 28 (47), pp. 103-113.

Robbins, S. \& Coulter, M. (2005). Administración. México: Pearson Educación.

Robbins, S.; Judge, T. (2008). Organizational Behavior. (s.c): Prentice Hall, $13 e d$.

Sanz de Santamaría, A. (2010). La educación y la gerencia humanista, charla dictada como parte de la cátedra Gerencia Humanista. Bogotá: Universidad EAN.

Schumacher, E.F. (1975). Small is Beautiful. Economics as if People Mattered. Harper and Row.

Scott, J.T. (2010). The Sustainable Business. European Foundation for Management Development.

Senge P.; Kleiner, A.; Roberts, Ch.; Ross, R. \& Smith, B. (1994). The Fifth Discipline Fieldbook. Currency Doubleday.

Senge, P. (2005). La Quinta Disciplina. España:Granica.

Shaw, G.; Brown, R.; Bromiley (1998). Strategic Stories: How 3M is Rewriting Business Planning, Harvard Business Review on Advances in Strategy. HBP. 
Spitzeck, H.; Pirson, M.; Amann, W.; Khan, S. y von Kimakowitz, E. (2009). Humanism in Business. New York: Cambridge University Press

Taylor, F. (1963). Principios de la administración científica. México: Herrero Hermanos.

Von Kimakowitz, E.; Spitzeck, H.; Pirson, M. \& Amann, W. (2011). Humanistic Management in Practice (s.c): Palgrave Macmillan.

United Nations Global Compact. (s.f). Los diez principios del pacto mundial. (s.c), (s.e).

Wei-ping, W.; Yuan-Duen, L. (2001). Participatory management and industrial relations climate: a study of Chinese, Japanese and US firms in Taiwan. The International Journal of Human Resource Management, 12 (5), pp. 827-844.

Wheatley, M. (1992). Leadership and the New Science. Learning About Organizations From an Orderly Universe. NY: Berrett-Koehler Publishers.

White, M.; Marsh, E. (2006). Content Analysis: A Flexible Methodology. Library Trends, 55, (1), pp. 22-45.

World Business Council for Sustainable Development (WBCSD) (2010). Vision 2050: una nueva agenda para los Negocios. (s.c), (s.e). 